\title{
Fault Identification Method of Diesel Engine in Light of Pearson Correlation Coefficient Diagram and Orthogonal Vibration Signals
}

\author{
Li Zhiyong $\left(\mathbb{D},{ }^{1,2,3}\right.$ Zhao Hongdong $\left(\mathbb{D},{ }^{1}\right.$ Zeng Ruili $\left(\mathbb{D},{ }^{4}\right.$ Xia Kewen $\mathbb{D}^{1},{ }^{1}$ \\ Guo Qiang, ${ }^{2}$ and Li Yuhai ${ }^{3}$ \\ ${ }^{1}$ School of Electronic and Information Engineering, Hebei University of Technology, Tianjin 300401, China \\ ${ }^{2}$ Department of Basic Science, Army Military Transportation University, Tianjin 300161, China \\ ${ }^{3}$ Key Laboratory of Electrooptical Information Control and Security Technology, Tianjin 300308, China \\ ${ }^{4}$ Department of Military Vehicle, Army Military Transportation University, Tianjin 300161, China
}

Correspondence should be addressed to Zhao Hongdong; zhaohd@hebut.edu.cn

Received 10 October 2018; Accepted 10 February 2019; Published 27 February 2019

Academic Editor: Pawel Packo

Copyright (C) 2019 Li Zhiyong et al. This is an open access article distributed under the Creative Commons Attribution License, which permits unrestricted use, distribution, and reproduction in any medium, provided the original work is properly cited.

\begin{abstract}
In order to select fault feature parameters simply and quickly and improve the identification rate of diesel engine faults by using the vibration signals, this paper proposes a diesel engine fault identification method on the basis of the Pearson correlation coefficient diagram (PCC Diagram) and the orthogonal vibration signals. At first, the orthogonal vibration acceleration signals are synchronously acquired in the direction of the top and side of the cylinder head. And the time-domain feature parameters are extracted from the orthogonal vibration acceleration signals to obtain the Pearson correlation coefficient (PCC). Then, the correlation coefficient diagram used to do feature parameter screening is constructed by selecting the feature parameters with the correlation coefficient of more than 0.9 . Finally, generalized regression neural network (GRNN) is adopted to classify and identify fuel supply fault in diesel engine. The results show that using the PCC Diagram can simplify the selection process of the feature parameters of the orthogonal vibration signals quickly and effectively. It can also improve the fault identification rate of diesel engine significantly with the help of adding the newly proposed cross-correlation coefficient of the orthogonal vibration signals into the GRNN input feature vector set.
\end{abstract}

\section{Introduction}

In accordance with the statistics by Sohu Auto and Ipsos in recent years, from the point of view of automotive faults, the number of faults occurring in the engine system accounts for more than $30 \%$ of the total with the highest proportion [1], thus causing great difficulties for production and life. So, some scholars have carried out a lot of researches, like collecting, extracting, and recognizing various kinds of information that appears during the working process of engines. Many engine fault detection and identification methods have been proposed, in which the most frequently used one is the using of the vibration signals [2-4]. By installing a vibration sensor somewhere in the engine to collect the corresponding vibration signal in the engine during operation, the collected vibration signal is processed in the time domain, frequency domain, or time-frequency domain, to further achieve the identification of specific faults at the location. Although this method obtains a relatively high fault identification rate, it often uses vibration signal in a single direction to detect and identify a particular fault. Since the engine is a reciprocating circulation machine, the mixture of gas in the cylinder is isotropic, and the gas pressure formed in the combustion process acts on the top and side of the cylinder head simultaneously $[5,6]$. That is, the vibration signals are generated on the surface of the cylinder head in the vertical and horizontal directions at the same time. Therefore, the vibration characteristics of the cylinder head in the vertical and horizontal directions should be taken into consideration in assessing of the working state of the 


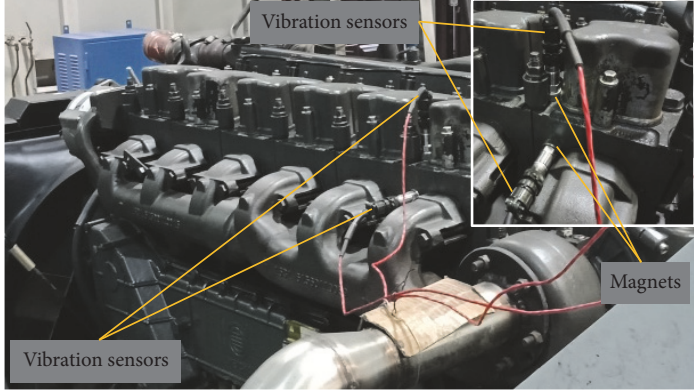

(a) Vibration sensors and magnets

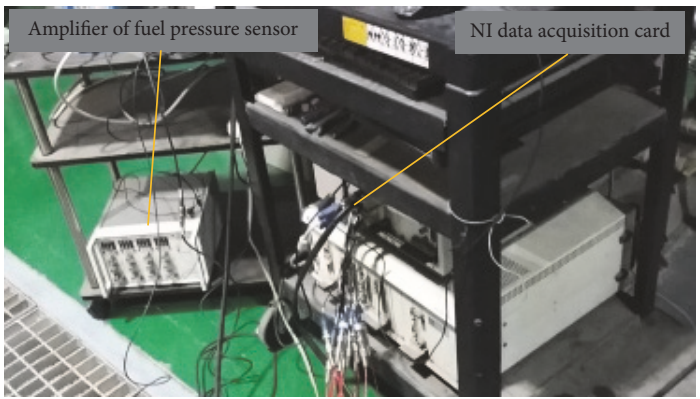

(b) Amplifier and data acquisition card

FIgURE 1: Sensors and the key setup.

engine. In addition, by the calculation of some experimental data, the correlation of the corresponding time-domain feature parameters of the orthogonal vibration acceleration signals acquired synchronously in the vertical direction of the cylinder head and horizontal direction of the cylinder head is proven to be small. That is to say, the corresponding time-domain feature parameters of the orthogonal vibration acceleration signals have weak correlation. So, this pair of orthogonal signals can be considered into use at the same time. Based on this, the PCC Diagram is adopted to optimize the orthogonal vibration signal feature selection to improve the fault identification rate of diesel engine.

Taking Weichai WD615 diesel engine as the research object, the paper carries out experimental researches on the faults of the fuel supply system for common engine faults. In the experiments, the engine fuel supply system faults are characterized by the fuel leakage of the sixth-cylinder highpressure fuel pipe under different severities. The experiments are conducted under four different working conditions: normal fuel supply, slight fuel leakage, severe fuel leakage, and fuel cut-off. The two vibration acceleration sensors are installed on the top and side of the cylinder head, which are perpendicular to each other. And the pair of orthogonal vibration signals on the surface of the cylinder head is synchronously collected. In order to avoid relatively complex calculations and improve the real-time performance of signal processing, only the time-domain feature parameters of the experimental data are extracted. The PCC are calculated after normalization, and the feature parameters with the correlation coefficient of more than 0.9 are selected to construct the coefficient diagram to screen feature parameters. Finally, the GRNN is adopted for fault classification and identification to verify the actual application effect of the PCC Diagram in identifying diesel engine faults with the orthogonal vibration signals.

\section{PCC}

The correlation coefficient is a basic method to reduce the dimensionality of high-dimensional data, and the PCC [7-9] is a more commonly used one. The PCC, proposed by Pearson in 1895 , is based on statistics. The mathematical calculation process of it is simple and accurate, and it can measure the linear correlation between variables well.

The PCC of variables (which can also be vectors or matrices) $X$ and $Y$ can be calculated by the following equation.

$$
\begin{aligned}
r_{\mathrm{XY}} & =\frac{\operatorname{cov}(X, Y)}{\sqrt{D(X)} \sqrt{D(Y)}} \\
& =\frac{\sum_{i=1}^{n}\left(x_{i}-\bar{x}\right)\left(y_{i}-\bar{y}\right)}{\sqrt{\sum_{i=1}^{n}\left(x_{i}-\bar{x}\right)^{2}} \sqrt{\sum_{i=1}^{n}\left(y_{i}-\bar{y}\right)^{2}}}
\end{aligned}
$$

In (1), $\operatorname{cov}(X, Y)$ is the covariance of $X$ and $Y ; D(X)$ is the variance of $X$, and $D(X)>0 ; D(Y)$ is the variance of $Y$, and $D(Y)>0 ; \bar{x}$ and $\bar{y}$ are the arithmetic mean of $X$ and $Y$, respectively. The range of the value of $r_{\mathrm{XY}}$ is $[-1,1]$. The larger the value is, the higher the linear correlation between $X$ and $Y$ is. When $r_{\mathrm{XY}}=1, X$ and $Y$ are completely positively correlated. When $r_{\mathrm{XY}}=-1, X$ and $Y$ are completely negatively correlated. When $r_{\mathrm{XY}}=0, X$ and $Y$ are independent.

\section{Feature Parameter Extraction and Fault Identification Procedure}

3.1. Experimental Data Acquisition. Weichai WD615 diesel engine assembled on Steyr truck is taken as the research object. It is an inline six-cylinder and water-cooled direct injection diesel engine with fuel injection pressure of 22.5 $\mathrm{MPa}$, fuel supply advance angle of $19-3^{\circ} \mathrm{CA}$, and rated speed of $2600 \mathrm{r} / \mathrm{min}$.

Multichannel sensors are installed in the sixth-cylinder of the diesel engine, as shown in Figure 1. Two vibration acceleration sensors, PCB M603C01, are attached to the top and side of the cylinder head with powerful magnets. They are used to achieve the detection of the vibration acceleration at the top and side of the cylinder head. The fuel pressure sensor is fixed on the high-pressure fuel pipe by a clamp, with a dedicated charge amplifier, to collect the fuel pressure and fuel supply time of the high-pressure fuel pipe. The Hall sensor is fixed on the flywheel housing to gather the rotational speed signal.

When the engine is running in no-load, the speed of the engine is set to $800 \mathrm{r} / \mathrm{min}$. And the host computer programs 
are written in Labview to drive the data acquisition card to collect the signals. The sampling frequency is $65536 \mathrm{~Hz}$.

Under different working conditions of the sixth-cylinder high-pressure fuel pipe, the speed signal, fuel pressure signal of the high-pressure fuel pipe, and vibration acceleration signals of the top and side of the cylinder head are simultaneously acquired via multiple sensors. 77 sets of data are collected from each channel under the condition of normal fuel supply, 62 sets of data under the condition of slight fuel leakage, 102 sets of data under the condition of severe fuel leakage, and 50 sets of data under the condition of fuel cutoff.

3.2. Feature Parameter Extraction. A complete combustion cycle of the diesel engine corresponds to a crankshaft angle range of $-360^{\circ} \mathrm{CA}-360^{\circ} \mathrm{CA}$. Weichai WD615 used in the experiment is a six-cylinder diesel engine with the firing sequence of 1-5-3-6-2-4. So, for the sixth-cylinder combustion, the corresponding crankshaft angle range is $1 / 6$ of a complete combustion cycle. That is, the crankshaft angle range is $60^{\circ} \mathrm{CA}$ to the left and to the right of the top dead center (TDC) of the sixth-cylinder, with $120^{\circ} \mathrm{CA}$ in total. The TDC of the sixth-cylinder is calibrated by each set of fuel pressure and rotation speed signals simultaneously captured under each of the above working conditions. Taking the sixth-cylinder TDC as reference, vibration acceleration signals that are synchronously acquired in the direction of the top and side of the cylinder head date with the same number of sampling points are extracted within the crank angle range of $-90^{\circ} \mathrm{CA}$ $90^{\circ} \mathrm{CA}$. The following 19 time-domain feature parameters are extracted: peak value, absolute peak value, peak-to-peak value, mean value, absolute mean value, root-mean-square (RMS) value, variance, standard deviation, root amplitude, Kurtosis index, Skewness index, peak index, waveform index, pulse index, margin index, peak value of autocorrelation function, peak value of cross-correlation function between all signals under different working conditions and normal fuel supply signal, peak correlation value between the vibration signal at the top of the cylinder head and the corresponding vibration signal at the side of the cylinder head of each working condition, and peak-to-peak correlation value between the vibration signal at the top of the cylinder head and the corresponding vibration signal at the side of the cylinder head of each working condition. The formulas for calculating these time-domain feature parameters are shown in Table 1 . The last three parameters are calculated by the same formula, but the input signals are different. The 19 time-domain feature parameters are represented by $F_{1}$ to $F_{19}$, respectively.

3.3. GRNN-Based Fault Identification Process. GRNN [1012 ] is a kind of feedforward neural network with mentors learning, belonging to radial basis function neural network (RBF). Because of the advantages of strong nonlinear mapping ability, good local approximation ability, fast learning speed, simple parameter adjustment during programming, good generalization ability, and good robustness, GRNN has been widely used in engineering.
TABLE 1: Formulas for time-domain feature parameters.

\begin{tabular}{c}
\hline Time-domain Feature Parameters $F_{1} \sim F_{17}$ \\
$F_{1}=\max \left(x_{i}\right)$ \\
$F_{2}=\max \left|x_{i}\right|$ \\
$F_{3}=\max \left(x_{i}\right)-\min \left(x_{i}\right)$ \\
$F_{4}=\frac{1}{N} \sum_{i=1}^{n} x_{i}$ \\
$F_{5}=\frac{1}{N} \sum_{i=1}^{n}\left|x_{i}\right|$ \\
$F_{6}=\sqrt{\frac{1}{N} \sum_{i=1}^{N} x_{i}^{2}}$ \\
$\frac{1}{N} \sum_{i=1}^{N}\left(x_{i}-\bar{x}\right)^{2}$ \\
$F_{8}=\sqrt{\frac{1}{N} \sum_{i=1}^{N}\left(x_{i}-\bar{x}\right)^{2}}$ \\
$F_{9}=\left(\frac{1}{N} \sum_{i=1}^{N} \sqrt{\left|x_{i}\right|}\right)^{2}$ \\
$F_{10}=\frac{E(x-\mu)^{4}}{\sigma^{4}}$ \\
$F_{11}=\frac{E(x-\mu)^{3}}{\sigma_{17}^{3}}$ \\
$F_{16}=\sum_{n=-\infty}^{\infty} x(n) y(n+m)$ \\
$F_{12}^{\infty} F_{13}=\frac{F_{6}}{F_{5}}$ \\
$F_{14}=\frac{F_{2}}{F_{5}}$ \\
$F_{9}$
\end{tabular}

The process of fuel supply fault identification by GRNN is shown in Figure 2. In the first step, the vibration acceleration signals of the top and side of the cylinder head are collected by the vibration acceleration sensors, respectively. Taking the TDC of the sixth-cylinder as the reference, the signal data are extracted when the crankshaft angle range is $90^{\circ} \mathrm{CA}-90^{\circ} \mathrm{CA}$. The second step is to extract the time-domain feature parameters from $F_{1}$ to $F_{17}$ of the two vibration signals and the time-domain feature parameters $F_{18}$ and $F_{19}$ of the orthogonal vibration signals. For the third step, the PCC Diagrams are, respectively, constructed for the vibration acceleration signals of the top and side of the cylinder head with the feature parameters whose correlation coefficient are more than 0.9. And the respective feature parameters $F_{1}$ to $F_{17}$ are screened. At the fourth step, there are eight conditions as follows: the use of the vibration signal at the top of the cylinder head, the use of the PCC Diagram to optimize the vibration signal at the top of the cylinder head, the use of the vibration signal at the side of the cylinder head, the use of the PCC Diagram to optimize the vibration signal at the side of the cylinder head, the use of the cylinder head orthogonal vibration signals directly, the use of the PCC Diagram to 


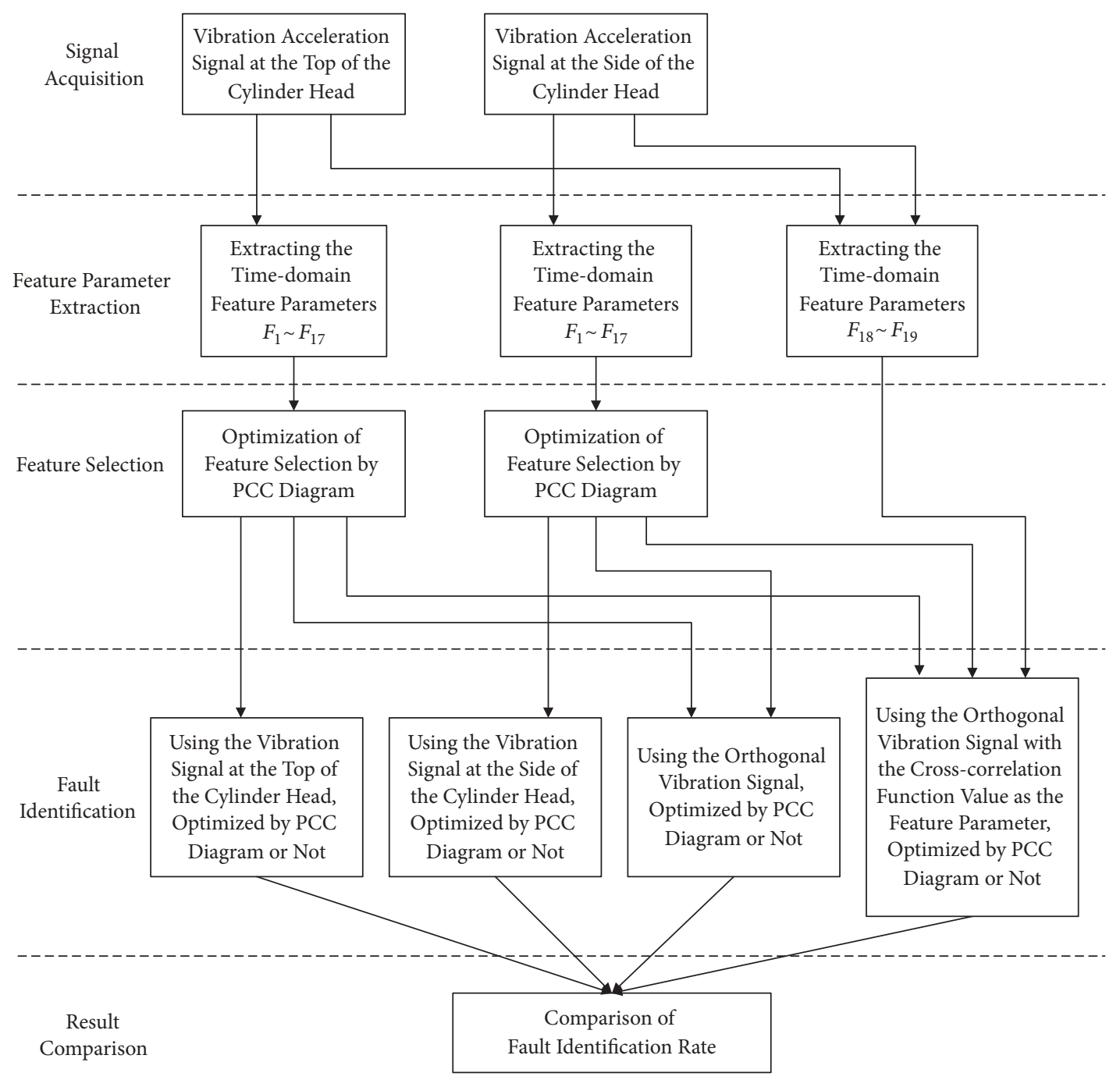

FIGURE 2: Flow of fuel supply fault identification by GRNN.

optimize the cylinder head orthogonal vibration signals, the use of the cylinder head orthogonal vibration signals and the introduction of the cross-correlation of the orthogonal vibration signals as the feature parameter, and the use of the PCC Diagram to optimize the orthogonal vibration signals of the cylinder head by introducing the cross-correlation of the orthogonal vibration signals as the special feature parameter. Under the eight conditions, the fault feature vector sets are constructed by using the corresponding time-domain feature parameters as the input of GRNN to identify the fault. In the fifth step, the advantages and disadvantages of the abovementioned various fault identification methods are compared to choose the method with the highest fault identification rate.

\section{PCC Diagram}

Under the condition of normal fuel supply of the highpressure fuel pipe, 20 sets of the orthogonal vibration acceleration signals synchronously collected at the top and side of the cylinder head are randomly selected to discuss their correlation.

4.1. Correlation of the Orthogonal Vibration Signals. As mentioned above, the orthogonal vibration signals at the top and side of the cylinder head should be taken into account simultaneously when evaluating the working state of the engine. Each time-domain feature parameter $F_{\mathrm{i}}(i=1 \sim 17)$ of the vibration signal at the top of the cylinder head is normalized in the selected 20 sets of data. Similarly, each time-domain feature parameter $F_{\mathrm{j}}(j=1 \sim 17)$ of the vibration signal at the side of the cylinder head is normalized in the selected 20 sets of data. The PCC of each time-domain feature parameter $F_{\mathrm{i}}$ of the vibration signal at the top of the cylinder head and each time-domain feature parameter $F_{\mathrm{j}}(i=j)$ of the corresponding vibration signal at the side of the cylinder head are obtained, as shown in Table 2.

It is not difficult to find that the PCC of the corresponding time-domain feature parameters of the orthogonal vibration acceleration signals at the top and side of the cylinder head are 
TABLE 2: Correlation coefficient of the orthogonal vibration signals.

\begin{tabular}{lc}
\hline Feature Parameter & Correlation Coefficient \\
\hline$F_{1}$ & 0.117718 \\
$F_{2}$ & 0.289401 \\
$F_{3}$ & 0.372678 \\
$F_{4}$ & 0.509780 \\
$F_{5}$ & 0.420775 \\
$F_{6}$ & 0.499927 \\
$F_{7}$ & 0.479980 \\
$F_{8}$ & 0.499932 \\
$F_{9}$ & 0.198451 \\
$F_{10}$ & 0.025351 \\
$F_{11}$ & 0.205991 \\
$F_{12}$ & -0.031970 \\
$F_{13}$ & 0.352877 \\
$F_{14}$ & 0.078338 \\
$F_{15}$ & 0.142896 \\
$F_{16}$ & 0.479969 \\
$F_{17}$ & 0.233233 \\
\hline
\end{tabular}

basically all below 0.5 . That is, the linear correlation is weak overall. So, this pair of orthogonal signals can be considered at the same time for analysis. Though the conclusion is obtained by the 20 sets of data selected randomly, the correctness of the conclusion has been confirmed by the entire dataset.

\subsection{PCC Diagram of Vibration Signal at Cylinder Head Top.} Dimensional reduction in high-dimensional data relying on the PCC can be accomplished by calculating the linear correlation coefficient between variables.

However, the linear correlation between multiple variables is often complex and reticular. Therefore, when filtering the feature parameters through the PCC, the feature parameters cannot simply be selected directly according to the value of the correlation coefficient. In this paper, the concept of correlation coefficient diagram is proposed. The correlation coefficient diagram is established by selecting the feature parameters with certain correlation. And then the selection of feature parameters is carried out in combination with Graph Theory.

Each time-domain feature parameter $F_{\mathrm{i}}(i=1 \sim 17)$ of the vibration signal at the top of the cylinder head is normalized among the 20 sets of data selected above, and the PCC between each other is calculated. That is, the PCC between $F_{\mathrm{i}}$ and $F_{\mathrm{j}}(i, j=1 \sim 17, i \neq j)$ is calculated. Selecting the feature parameters with the correlation coefficient of more than 0.9 (the threshold value 0.9 is selected by experimental data) and connecting them to each other with line segment, a correlation network graph is formed, that is, the PCC Diagram, as shown in Figure 3. The correlation coefficient diagram obtained in this way is an undirected graph. Each node of the graph represents a time-domain feature parameter, and each line of the graph represents the correlation of two connected time-domain feature parameters with the correlation coefficient of more than 0.9.
TABLE 3: Combinations of cylinder head top signal feature parameters.

\begin{tabular}{lc}
\hline Reserved Feature Parameters & $\begin{array}{c}\text { Removed Feature } \\
\text { Parameters }\end{array}$ \\
\hline$F_{1} \sim F_{6}, F_{9} \sim F_{13}, F_{17}$ & $F_{7} \sim F_{8}, F_{14} \sim F_{16}$ \\
$F_{1} \sim F_{5}, F_{7}, F_{9} \sim F_{13}, F_{17}$ & $F_{6}, F_{8}, F_{14} \sim F_{16}$ \\
$F_{1} \sim F_{5}, F_{8} \sim F_{13}, F_{17}$ & $F_{6} \sim F_{7}, F_{14} \sim F_{16}$ \\
$F_{1} \sim F_{5}, F_{9} \sim F_{13}, F_{16} \sim F_{17}$ & $F_{6} \sim F_{8}, F_{14} \sim F_{15}$ \\
$F_{1}, F_{3} \sim F_{6}, F_{9} \sim F_{11}, F_{13}, F_{15}, F_{17}$ & $F_{2}, F_{7} \sim F_{8}, F_{12}, F_{14}, F_{16}$ \\
$F_{1}, F_{3} \sim F_{5}, F_{7}, F_{9} \sim F_{11}, F_{13}, F_{15}$, & $F_{2}, F_{6}, F_{8}, F_{12}, F_{14}, F_{16}$ \\
$F_{17}$ & $F_{2}, F_{6} \sim F_{7}, F_{12}, F_{14}, F_{16}$ \\
$F_{1}, F_{3} \sim F_{5}, F_{8} \sim F_{11}, F_{13}, F_{15}, F_{17}$ & $F_{2}, F_{6} \sim F_{8}, F_{12}, F_{14}$ \\
$F_{1}, F_{3} \sim F_{5}, F_{9} \sim F_{11}, F_{13}, F_{15} \sim F_{17}$ & $F_{2}, F_{7} \sim F_{8}, F_{12}, F_{15} \sim F_{16}$ \\
$F_{1}, F_{3} \sim F_{6}, F_{9} \sim F_{11}, F_{13} \sim F_{14}, F_{17}$ & $F_{2}, F_{6}, F_{8}, F_{12}, F_{15} \sim F_{16}$ \\
$F_{1}, F_{3} \sim F_{5}, F_{7}, F_{9} \sim F_{11}, F_{13} \sim F_{14}$, & $F_{2}, F_{6} \sim F_{7}, F_{12}, F_{15} \sim F_{16}$ \\
$F_{17}$ & $F_{2}, F_{6} \sim F_{8}, F_{12}, F_{15}$ \\
$F_{1}, F_{3} \sim F_{5}, F_{8} \sim F_{11}, F_{13} \sim F_{14}, F_{17}$ & \\
$F_{1}, F_{3} \sim F_{5}, F_{9} \sim F_{11}, F_{13} \sim F_{14}$, & $F_{16} \sim F_{17}$
\end{tabular}

Combining with the related knowledge in Graph Theory, the reservation and removal methods of the feature parameters are analyzed to obtain linearly independent feature parameters. In Figure 3(a), if $F_{2}$ is reserved, $F_{14}$ and $F_{15}$ are removed, while $F_{12}$ is reserved, so that $F_{2}$ and $F_{12}$ are linearly independent of each other. If $F_{14}$ or $F_{15}$ is reserved, the other three feature parameters can be removed. The reservation and removal methods of $F_{2}, F_{12}, F_{14}$, and $F_{15}$ are listed using the combination method, and then three combinations can be attained. In Figure 3(b), the linear correlation between $F_{6}, F_{7}$, $F_{8}$, and $F_{16}$ is excellent, so three of them can be arbitrarily removed. For these four feature parameters, the combination method is used to enumerate the reservation and removal methods. There are four combinations in total. Then, in accordance with the combination method, the reservation and removal methods of the two sets of feature parameters $F_{2}, F_{12}, F_{14}, F_{15}$ and $F_{6}, F_{7}, F_{8}, F_{16}$ are enumerated. There are twelve combinations of the feature parameters reserved and removed from $F_{1}$ to $F_{17}$, as shown in Table 3.

4.3. PCC Diagram of Vibration Signal at Cylinder Head Side. Similarly, each time-domain feature parameter $F_{\mathrm{m}}(m=1 \sim$ 17) of the vibration signal at the side of the cylinder head is normalized among the above-mentioned 20 sets of data. And the PCC between each other is obtained. This is the PCC between $F_{\mathrm{m}}$ and $F_{\mathrm{n}}(m, n=1 \sim 17, m \neq n)$. Selecting the feature parameters with the correlation coefficient of more than 0.9 and connecting them to each other with line segment, a correlation network graph is formed, that is, the PCC Diagram, as shown in Figure 4. It is also an undirected graph. Each node of the graph represents a time-domain feature parameter, and each line of the graph represents the correlation of two connected time-domain feature parameters with the correlation coefficient of more than 0.9 . 


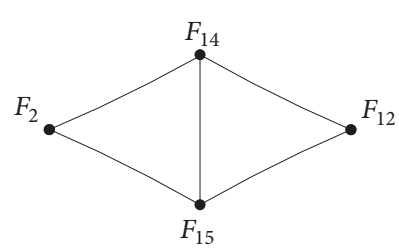

(a) Feature parameters $F_{2}, F_{12}$, $F_{14} \sim F_{15}$

FIGURE 3: PCC Diagram of cylinder head top signal.

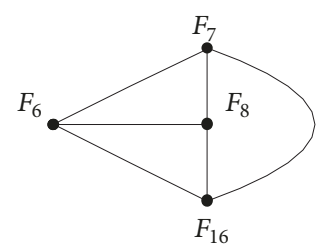

(b) Feature parameters $F_{6} \sim F_{8}, F_{16}$

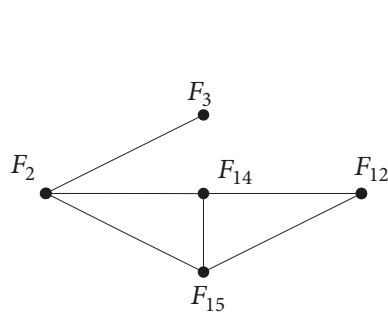

(a) Feature parameters $F_{2} \sim F_{3}$, $F_{12}, F_{14} \sim F_{15}$

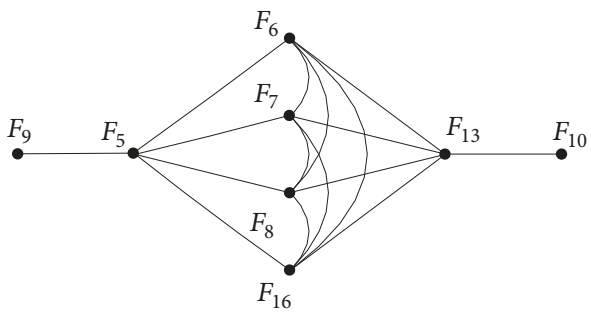

(b) Feature parameters $F_{5} \sim F_{10}, F_{13}, F_{16}$

FIGURE 4: PCC Diagram of cylinder head side signal.

The connections of the time-domain feature parameters in Figure 4(a) are similar to those in Figure 3(a). If $F_{2}$ is reserved in Figure $4(\mathrm{a})$, then $F_{3}, F_{14}$, and $F_{15}$ are removed while $F_{12}$ is reserved, so that $F_{2}$ and $F_{12}$ are linearly independent of each other. If $F_{14}$ is reserved, then $F_{2}, F_{12}$, and $F_{15}$ are removed, and $F_{3}$ and $F_{14}$ are linearly independent of each other. If $F_{15}$ is reserved, $F_{2}, F_{12}$, and $F_{14}$ are removed, and $F_{3}$ and $F_{15}$ are linearly independent. If $F_{12}$ is reserved, $F_{14}$ and $F_{15}$ are removed, and $F_{2}$ and $F_{12}$ are linearly independent of each other (already existing) or $F_{3}$ and $F_{12}$ are linearly independent of each other. The reservation and removal methods of $F_{2}, F_{3}, F_{12}, F_{14}$, and $F_{15}$ are listed using the combination method, and then four combinations can be attained. In Figure $4(\mathrm{~b})$, if $F_{5}$ is reserved, then $F_{6}, F_{7}, F_{8}, F_{9}$, and $F_{16}$ are removed, and $F_{5}$ and $F_{10}$ are linearly independent of each other or $F_{5}$ and $F_{13}$ are linearly independent of each other. If any one of $F_{6}, F_{7}, F_{8}$, or $F_{16}$ is reserved, the other three will be removed at the same time with $F_{5}$ and $F_{13}$, and the reserved feature parameter is linearly independent from $F_{9}$ and $F_{10}$, and there are four combinations in total. If $F_{13}$ is reserved, then $F_{6}, F_{7}, F_{8}, F_{10}$, and $F_{16}$ are removed, and $F_{5}$ and $F_{13}$ are linearly independent of each other (already existing) or $F_{9}$ and $F_{13}$ are linearly independent of each other. The reservation and removal methods of $F_{5}, F_{6}, F_{7}, F_{8}, F_{9}$, $F_{10}, F_{13}$, and $F_{16}$ are listed using the combination method, and there are seven combinations in total. Then, the combination method is used to enumerate the reservation and removal methods of the two sets of feature parameters between $F_{2}$, $F_{3}, F_{12}, F_{14}, F_{15}$ and $F_{5}, F_{6}, F_{7}, F_{8}, F_{9}, F_{10}, F_{13}, F_{16}$. So, there are 28 combinations of the feature parameters reserved and removed from $F_{1}$ to $F_{17}$ in total, as shown in Table 4 .

\section{Fuel Supply Fault Identification}

In consideration of the conditions of normal fuel supply, slight fuel leakage, severe fuel leakage, and fuel cut-off at the same time, the fuel supply fault identification results for various conditions are shown in Figure 5. The data for GRNN under different working conditions are divided as shown in Table 5.

As for the distribution of fault identification results, most of the existing articles use planar graphs (two-dimensional graphs). But, the display method is not intuitive and the effect is very general. Figure 5 shows the distribution of fault identification results more intuitively from the perspective of three-dimensional space.

When using GRNN to identify the diesel engine faults, the time-domain feature parameters of the vibration signal at the top of the cylinder head from $F_{1}$ to $F_{17}$ are used as the input of GRNN. And the result of diesel engine fault identification by using the vibration signal at the top of the cylinder head can be obtained, as shown in Figure 5(a). The result of diesel engine fault identification by using the vibration signal at the side of the cylinder head can be obtained by taking the time-domain feature parameters of the vibration signal at the side of the cylinder head from $F_{1}$ to $F_{17}$ as the input of GRNN, as shown in Figure 5(b). As mentioned earlier, the linear correlation of the corresponding time-domain feature parameters of the orthogonal vibration acceleration signals at the top and side of the cylinder head is weak overall. So, when using this pair of orthogonal vibration signals to identify the diesel fault, the corresponding time-domain feature parameters of the pair of orthogonal vibration signals 
TABLE 4: Combinations of cylinder head side signal feature parameters.

\begin{tabular}{|c|c|}
\hline Reserved Feature Parameters & Removed Feature Parameters \\
\hline$F_{1} \sim F_{2}, F_{4} \sim F_{5}, F_{10} \sim F_{12}, F_{17}$ & $F_{3}, F_{6} \sim F_{9}, F_{13} \sim F_{16}$ \\
\hline$F_{1} \sim F_{2}, F_{4} \sim F_{5}, F_{11} \sim F_{13}, F_{17}$ & $F_{3}, F_{6} \sim F_{10}, F_{14} \sim F_{16}$ \\
\hline$F_{1} \sim F_{2}, F_{4}, F_{6}, F_{9} \sim F_{12}, F_{17}$ & $F_{3}, F_{5}, F_{7} \sim F_{8}, F_{13} \sim F_{16}$ \\
\hline$F_{1} \sim F_{2}, F_{4}, F_{7}, F_{9} \sim F_{12}, F_{17}$ & $F_{3}, F_{5} \sim F_{6}, F_{8}, F_{13} \sim F_{16}$ \\
\hline$F_{1} \sim F_{2}, F_{4}, F_{8} \sim F_{12}, F_{17}$ & $F_{3}, F_{5} \sim F_{7}, F_{13} \sim F_{16}$ \\
\hline$F_{1} \sim F_{2}, F_{4}, F_{9} \sim F_{12}, F_{16} \sim F_{17}$ & $F_{3}, F_{5} \sim F_{8}, F_{13} \sim F_{15}$ \\
\hline$F_{1} \sim F_{2}, F_{4}, F_{9}, F_{11} \sim F_{13}, F_{17}$ & $F_{3}, F_{5} \sim F_{8}, F_{10}, F_{14} \sim F_{16}$ \\
\hline$F_{1}, F_{3} \sim F_{5}, F_{10} \sim F_{12}, F_{17}$ & $F_{2}, F_{6} \sim F_{9}, F_{13} \sim F_{16}$ \\
\hline$F_{1}, F_{3} \sim F_{5}, F_{11} \sim F_{13}, F_{17}$ & $F_{2}, F_{6} \sim F_{10}, F_{14} \sim F_{16}$ \\
\hline$F_{1}, F_{3} \sim F_{4}, F_{6}, F_{9} \sim F_{12}, F_{17}$ & $F_{2}, F_{5}, F_{7} \sim F_{8}, F_{13} \sim F_{16}$ \\
\hline$F_{1}, F_{3} \sim F_{4}, F_{7}, F_{9} \sim F_{12}, F_{17}$ & $F_{2}, F_{5} \sim F_{6}, F_{8}, F_{13} \sim F_{16}$ \\
\hline$F_{1}, F_{3} \sim F_{4}, F_{8} \sim F_{12}, F_{17}$ & $F_{2}, F_{5} \sim F_{7}, F_{13} \sim F_{16}$ \\
\hline$F_{1}, F_{3} \sim F_{4}, F_{9} \sim F_{12}, F_{16} \sim F_{17}$ & $F_{2}, F_{5} \sim F_{8}, F_{13} \sim F_{15}$ \\
\hline$F_{1}, F_{3} \sim F_{4}, F_{9}, F_{11} \sim F_{13}, F_{17}$ & $F_{2}, F_{5} \sim F_{8}, F_{10}, F_{14} \sim F_{16}$ \\
\hline$F_{1}, F_{3} \sim F_{5}, F_{10} \sim F_{11}, F_{14}, F_{17}$ & $F_{2}, F_{6} \sim F_{9}, F_{12} \sim F_{13}, F_{15} \sim F_{16}$ \\
\hline$F_{1}, F_{3} \sim F_{5}, F_{11}, F_{13} \sim F_{14}, F_{17}$ & $F_{2}, F_{6} \sim F_{10}, F_{12}, F_{15} \sim F_{16}$ \\
\hline $\begin{array}{l}F_{1}, F_{3} \sim F_{4}, F_{6}, F_{9} \sim F_{11}, F_{14}, \quad F \\
F_{17}\end{array}$ & $F_{2}, F_{5}, F_{7} \sim F_{8}, F_{12} \sim F_{13}, F_{15} \sim F_{16}$ \\
\hline $\begin{array}{l}F_{1}, F_{3} \sim F_{4}, F_{7}, F_{9} \sim F_{11}, F_{14}, \\
F_{17}\end{array}$ & $F_{2}, F_{5} \sim F_{6}, F_{8}, F_{12} \sim F_{13}, F_{15} \sim F_{16}$ \\
\hline$F_{1}, F_{3} \sim F_{4}, F_{8} \sim F_{11}, F_{14}, F_{17}$ & $F_{2}, F_{5} \sim F_{7}, F_{12} \sim F_{13}, F_{15} \sim F_{16}$ \\
\hline $\begin{array}{l}F_{1}, F_{3} \sim F_{4}, F_{9} \sim F_{11}, F_{14} \\
F_{16} \sim F_{17}\end{array}$ & $F_{2}, F_{5} \sim F_{8}, F_{12} \sim F_{13}, F_{15}$ \\
\hline $\begin{array}{l}F_{1}, F_{3} \sim F_{4}, F_{9}, F_{11}, F_{13} \sim F_{14}, \\
F_{17}\end{array}$ & $F_{2}, F_{5} \sim F_{8}, F_{10}, F_{12}, F_{15} \sim F_{16}$ \\
\hline$F_{1}, F_{3} \sim F_{5}, F_{10} \sim F_{11}, F_{15}, F_{17}$ & $F_{2}, F_{6} \sim F_{9}, F_{12} \sim F_{14}, F_{16}$ \\
\hline$F_{1}, F_{3} \sim F_{5}, F_{11}, F_{13}, F_{15}, F_{17}$ & $F_{2}, F_{6} \sim F_{10}, F_{12}, F_{14}, F_{16}$ \\
\hline $\begin{array}{l}F_{1}, F_{3} \sim F_{4}, F_{6}, F_{9} \sim F_{11}, F_{15}, \\
F_{17}\end{array}$ & $F_{2}, F_{5}, F_{7} \sim F_{8}, F_{12} \sim F_{14}, F_{16}$ \\
\hline $\begin{array}{l}F_{1}, F_{3} \sim F_{4}, F_{7}, F_{9} \sim F_{11}, F_{15}, \\
F_{17}\end{array}$ & $F_{2}, F_{5} \sim F_{6}, F_{8}, F_{12} \sim F_{14}, F_{16}$ \\
\hline$F_{1}, F_{3} \sim F_{4}, F_{8} \sim F_{11}, F_{15}, F_{17}$ & $F_{2}, F_{5} \sim F_{7}, F_{12} \sim F_{14}, F_{16}$ \\
\hline$F_{1}, F_{3} \sim F_{4}, F_{9} \sim F_{11}, F_{15} \sim F_{17}$ & $F_{2}, F_{5} \sim F_{8}, F_{12} \sim F_{14}$ \\
\hline $\begin{array}{l}F_{1}, F_{3} \sim F_{4}, F_{9}, F_{11}, F_{13}, F_{15} \\
F_{17}\end{array}$ & $F_{2}, F_{5} \sim F_{8}, F_{10}, F_{12}, F_{14}, F_{16}$ \\
\hline
\end{tabular}

TABLE 5: Data dividing for GRNN.

\begin{tabular}{lcc}
\hline Working condition & $\begin{array}{c}\text { Number of training } \\
\text { data }\end{array}$ & $\begin{array}{c}\text { Number of testing } \\
\text { data }\end{array}$ \\
\hline Normal fuel supply & 40 & 37 \\
Slight fuel leakage & 40 & 22 \\
Severe fuel leakage & 70 & 32 \\
Fuel cut-off & 30 & 20 \\
\hline
\end{tabular}

can be directly used as the input of GRNN at the same time. That is, the result of diesel engine fault identification can be directly obtained by using the orthogonal vibration signals of the cylinder head, as shown in Figure 5(c).
Furthermore, the feature parameters of the different combination methods reserved in Table 3 are used as the input of GRNN. The corresponding fault identification results are compared to find a feature parameter combination mode with the highest fault identification rate. That is the corresponding diesel engine fault identification rate of optimizing the selection of the feature parameters of the signal at the top of the cylinder head by using the PCC Diagram, as shown in Figure 5(d). Taking the feature parameters of the different combination methods reserved in Table 4 as the input of GRNN and comparing the corresponding fault identification results, the feature parameter combination mode with the highest fault identification rate is also found, that is, the corresponding diesel engine fault identification rate of optimizing the selection of the feature parameters of the signal at the side of the cylinder head by using the PCC Diagram, as shown in Figure 5(e). Taking the two sets of feature parameters by optimizing the selection of vibration signal feature parameters at the top and side of the cylinder head using the PCC Diagram as the input of GRNN, the corresponding fault identification results are compared to attain the combination of feature parameters with the highest fault identification rate, that is, the corresponding diesel engine fault identification rate of optimizing the selection of the feature parameters of the cylinder head orthogonal vibration signals by using the PCC Diagram, as shown in Figure 5(f).

Moreover, on the basis of identifying the diesel engine fault using the orthogonal vibration signals of the cylinder head directly, the orthogonal vibration signal crosscorrelation coefficient $F_{18}$ and $F_{19}$ are added as the input of GRNN. The corresponding fault identification results are compared to find the feature parameter combination with the highest fault identification rate, that is, the diesel engine fault identification rate of using the orthogonal vibration signals of the cylinder head and introducing the cross-correlation coefficient of the orthogonal vibration signals as the feature parameters, as shown in Figure 5(g). The diesel engine fault identification result of optimizing the orthogonal vibration signal feature parameters of the cylinder head by using the PCC Diagram and introducing the cross-correlation coefficient of the orthogonal vibration signals $F_{18}$ and $F_{19}$ as the feature parameters is shown in Figure 5(h).

It is not difficult to find that the graphical display method, like that in Figure 5, shows the distribution of fault identification results intuitively and clearly. At the same time, it can show the degree of dispersion of fault identification results intuitively and reflect the aesthetic feeling of natural science better.

Of course, in order to illustrate the results of fault identification more accurately with specific numerical values, Table 6 is introduced.

The results of fault identification are compared and analyzed in combination with Figure 5 and Table 6 as follows.

Figures 5(a), 5(b), and 5(c) are not using the feature parameter screening. All the feature parameters of each signal are directly used as the input of GRNN, and the diesel engine fault identification results are obtained. Compared with only using the vibration signal at the top of the cylinder head 

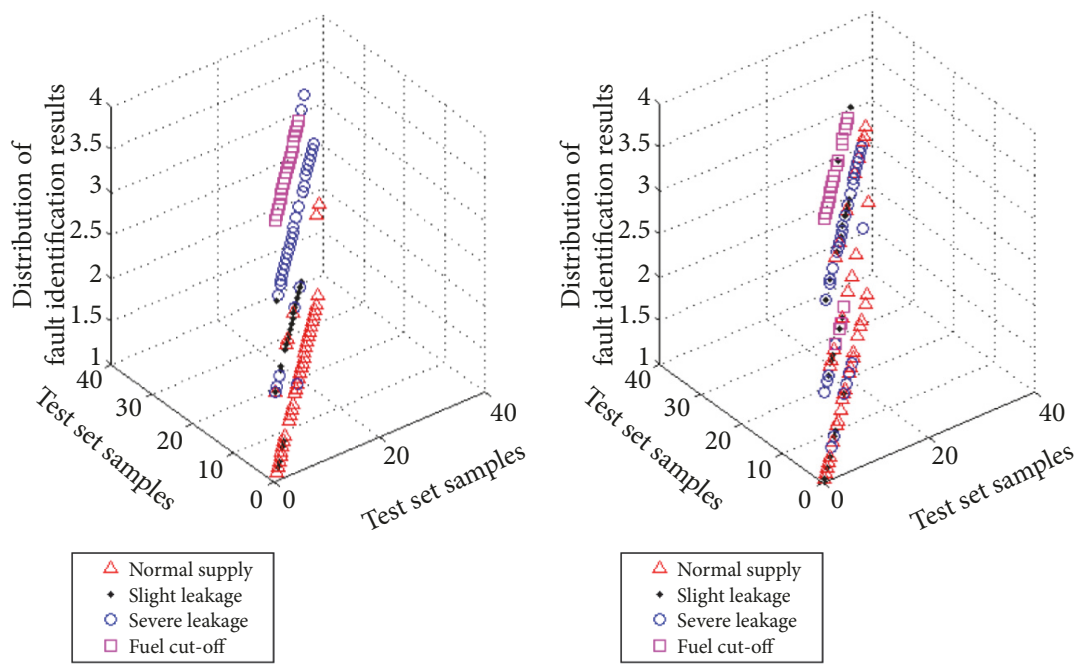

(a) Using vibration signal of cylinder head top

(b) Using vibration signal of cylinder head side
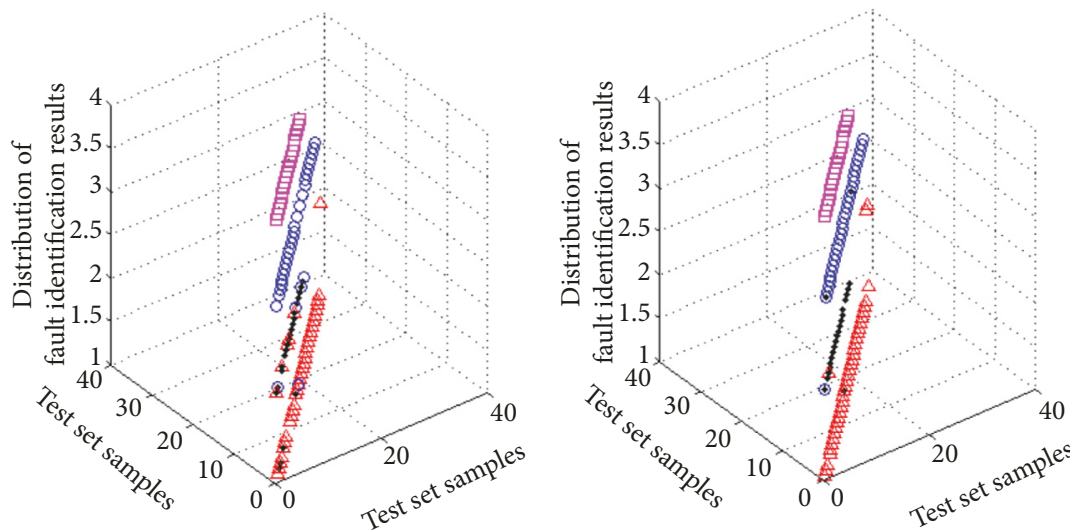

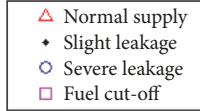

(c) Using orthogonal vibration signals

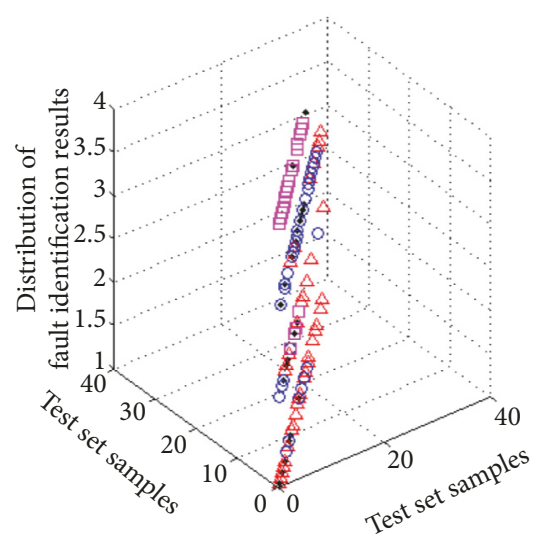

$$
\begin{aligned}
& \triangle \text { Normal supply } \\
& \text { - Slight leakage } \\
& \text { - Severe leakage }
\end{aligned}
$$

(e) Using vibration signal of cylinder head side, optimized by PCC Diagram

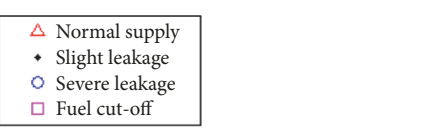

(d) Using vibration signal of cylinder head top, optimized by PCC Diagram

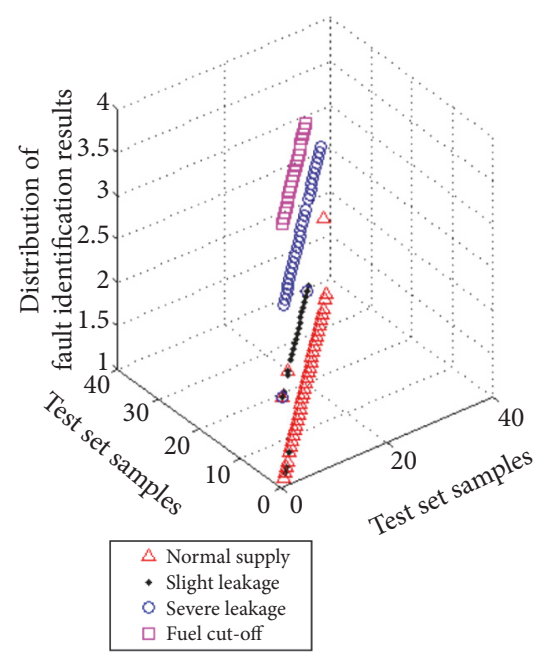

(f) Using orthogonal vibration signals, optimized by PCC Diagram

FIgUre 5: Continued. 

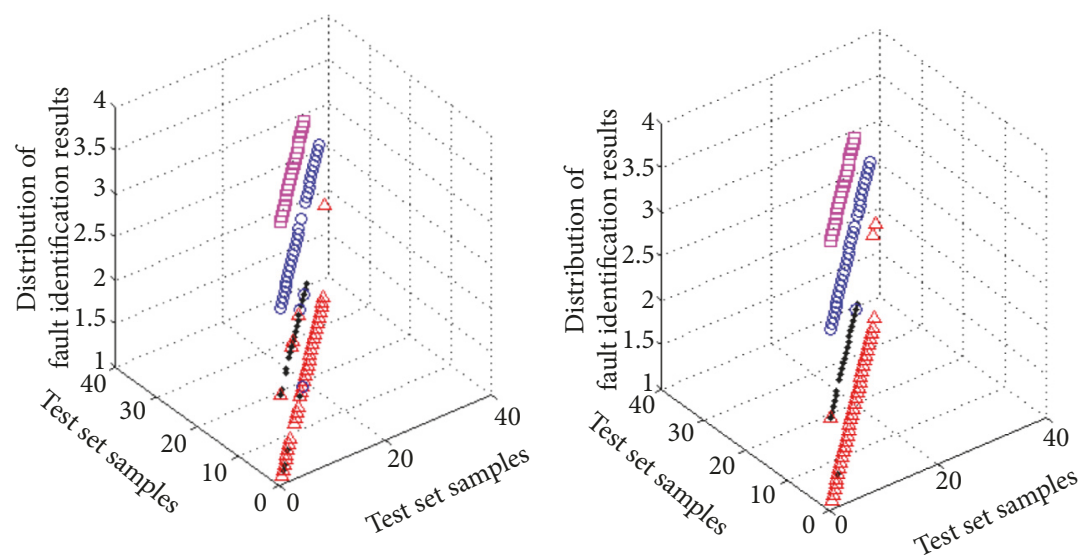

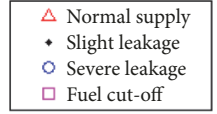

(g) Using orthogonal vibration signals, introducing cross-correlation coefficient

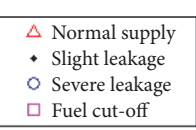

(h) Using orthogonal vibration signals, optimized by PCC Diagram, introducing crosscorrelation coefficient

FIGURE 5: Comparison of diesel engine fault identification results by GRNN.

TABLE 6: Identification rates in percent corresponding to Figure 5.

\begin{tabular}{|c|c|c|c|c|}
\hline \multirow{2}{*}{ Corresponding Figure } & \multicolumn{4}{|c|}{ Fault identification rate under different working conditions (\%) } \\
\hline & Normal fuel supply & Slight fuel leakage & Severe fuel leakage & Fuel cut-off \\
\hline Figure 5(a) & 83.78 & 77.27 & 75 & 100 \\
\hline Figure 5(b) & 56.76 & 22.73 & 65.63 & 80 \\
\hline Figure 5(c) & 83.78 & 81.82 & 84.38 & 100 \\
\hline Figure 5(d) & 91.89 & 86.36 & 96.88 & 100 \\
\hline Figure 5(e) & 56.76 & 22.73 & 65.63 & 80 \\
\hline Figure 5(f) & 91.89 & 86.36 & 93.75 & 100 \\
\hline Figure 5(g) & 86.49 & 81.82 & 90.63 & 100 \\
\hline Figure 5(h) & 91.89 & 95.45 & 96.88 & 100 \\
\hline
\end{tabular}

Note: the data of the table in bold are the highest fault identification rate obtained under different working conditions when using different fault identification methods.

(Figure 5(a)) or only using the vibration signal at the side of the cylinder head (Figure 5(b)), it is not difficult to find that the higher fault identification rate is relatively easily obtained by using the orthogonal vibration signals of the cylinder head (Figure 5(c)).

Respectively, comparing Figure 5(a) with Figure 5(d), Figure 5(b) with Figure 5(e), and Figure 5(c) with Figure 5(f), it can be found that after optimizing the vibration signals by using the PCC Diagram, the number of feature parameters is significantly reduced and the fault identification rates under the four working conditions are obviously improved. Moreover, relying on the PCC diagram to optimize the orthogonal vibration signals of the cylinder head can get a higher fault identification rate.

Comparing Figure 5(g) with Figures 5(a), 5(b), and 5(c), it is observed that the introduction of the orthogonal vibration signal cross-correlation coefficient as the feature parameters can obtain a higher fault identification rate under the four working conditions compared with only using vibration signal at the top (Figure 5(a)) and the side (Figure 5(b)) of the cylinder head and the orthogonal vibration signals of the cylinder head (Figure 5(c)).

Comparing Figures 5(h) and 5(g), it is found that after using the PCC Diagram to optimize the orthogonal vibration signals of the cylinder head and introducing the crosscorrelation coefficient of the orthogonal vibration signals as the feature parameters, the fault identification rates under the four working conditions are all up to over $90 \%$. They are $91.89 \%, 95.45 \%, 96.88 \%$, and $100 \%$, respectively. In comparison with the former methods for fault identification, this method can improve the fault identification rate to a greater extent and reduce the degree of fault identification dispersion of wrong results.

\section{Conclusion}

The experimental researches on a large number of fuel supply faults for Weichai WD615 diesel engine show that, on the 
premise of only the time-domain feature parameters, it is easy to obtain a higher fault identification rate by using the orthogonal vibration signals to identify faults in the diesel engine. The PCC Diagram can be used to simplify the feature parameter screening visually and vividly, thus improving the diesel engine fault identification rate to a greater extent. Furthermore, the cross-correlation coefficient of the orthogonal vibration signals is a very important time-domain feature parameter found in the orthogonal vibration tests, adding it into the feature vector set as the input of GRNN can obviously improve the fault identification rate of diesel engine by using the orthogonal vibration signals of the cylinder head. In particularly, after screening the feature parameters by using the coefficient diagram, the fault identification rates of more than $90 \%$ are obtained under all the working conditions. This fault identification method based on diesel engine can be applied to information extraction and fault identification for other reciprocating circulation machines.

\section{Data Availability}

The data used to support the findings of this study are available from the corresponding author upon request.

\section{Conflicts of Interest}

The authors declare that there are no conflicts of interest regarding the publication of this paper.

\section{Acknowledgments}

This paper is funded by Tactical Vehicle Status Monitoring, Fault Diagnosis and Information Management System Technology (40407030401, the Preresearch Project of the General Equipment Department of PLA) and the 13th FiveYear National Defense Science and Technology Key Laboratory Fund in 2017 (61421070104, the Research and Order Bureau of the Military Commission Equipment Development Department).

\section{References}

[1] M.-H.-N. Tayarani, X. Yao, and H. Xu, "Meta-heuristic algorithms in car engine design: A literature survey," IEEE Transactions on Evolutionary Computation, vol. 19, no. 5, pp. 609-629, 2015.

[2] I. Komorska, "Adaptive model of engine vibration signal for diagnostics of mechanical defects," Mechanika, vol. 19, no. 3, pp. 301-305, 2013.

[3] P. Czech and J. Mikulski, "Application of bayes classifier and entropy of vibration signals to diagnose damage of head gasket in internal combustion engine of a car," in Proceedings of International Conference on Transport Systems Telematics, vol. 471, pp. 225-232, Springer, Berlin, Germany, 2014.

[4] A. Puchalski, "A technique for the vibration signal analysis in vehicle diagnostics," Mechanical Systems and Signal Processing, vol. 56-57, pp. 173-180, 2015.

[5] G.-Q. Shu, J.-S. Wei, and H.-Q. Wei, "A numerical method to analyze thermoacoustics in an internal combustion engine by coupling wave equation," Journal of Combustion Science and Technology, vol. 16, no. 4, pp. 295-302, 2010.

[6] Y. H. Zhang, Combustion and explosion (Second Edition), Metallurgical Industry Press, Beijing, 2015.

[7] D. Li, X. Tang, C. Zhou, and K.-K. Phoon, "Uncertainty analysis of correlated non-normal geotechnical parameters using Gaussian copula," Science China Technological Sciences, vol. 55, no. 11, pp. 3081-3089, 2012.

[8] W. Yu, X. Yao, X. Liu, L. Li, and G. Zhai, "Ghost imaging based on Pearson correlation coefficients," Chinese Physics B, vol. 24, no. 5, pp. 340-345, 2015.

[9] W. Feng, Q. Zhu, J. Zhuang, and S. Yu, "An expert recommendation algorithm based on Pearson correlation coefficient and FP-growth," Cluster Computing, no. 3, pp. 1-12, 2018.

[10] J. Wiszniowski, "Applying the general regression neural network to ground motion prediction equations of induced events in the legnica-głogów copper district in Poland," Acta Geophysica, vol. 64, no. 6, pp. 2430-2448, 2016.

[11] R. Ahmadi, J. Shahrabi, and B. Aminshahidy, "Automatic well-testing model diagnosis and parameter estimation using artificial neural networks and design of experiments," Journal of Petroleum Exploration and Production Technology, vol. 7, no. 3, pp. 759-783, 2017.

[12] C.-S. Chen, C.-M. Weng, C.-J. Lin, and H.-W. Liu, "The use of a novel auto-focus technology based on a GRNN for the measurement system for mesh membranes," Microsystem Technologies, vol. 23, no. 2, pp. 343-353, 2017. 


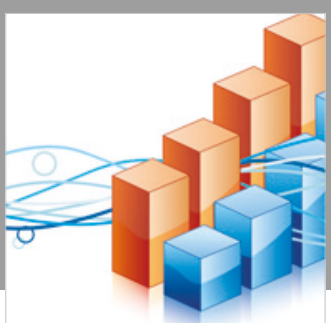

Advances in

Operations Research

\section{-n-m}
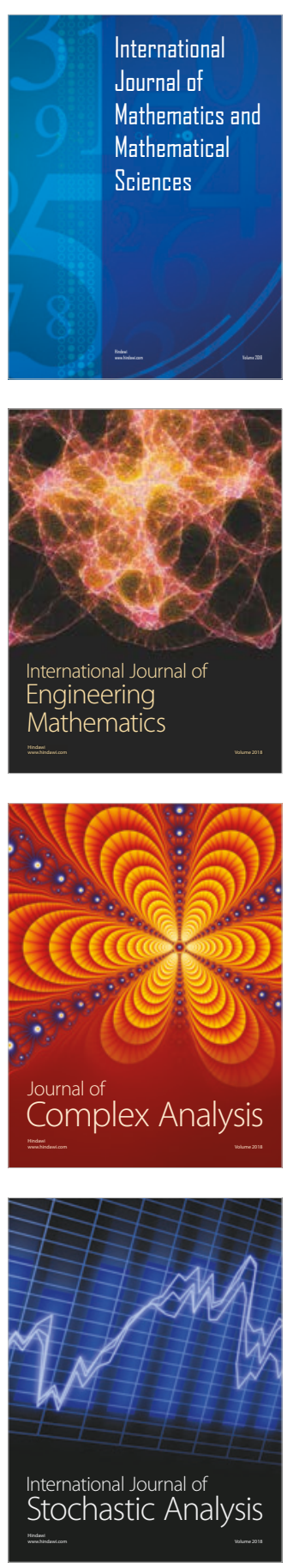
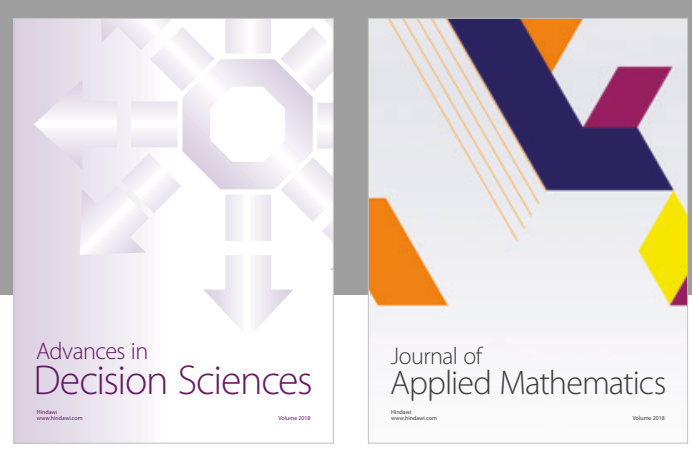

Journal of

Applied Mathematics
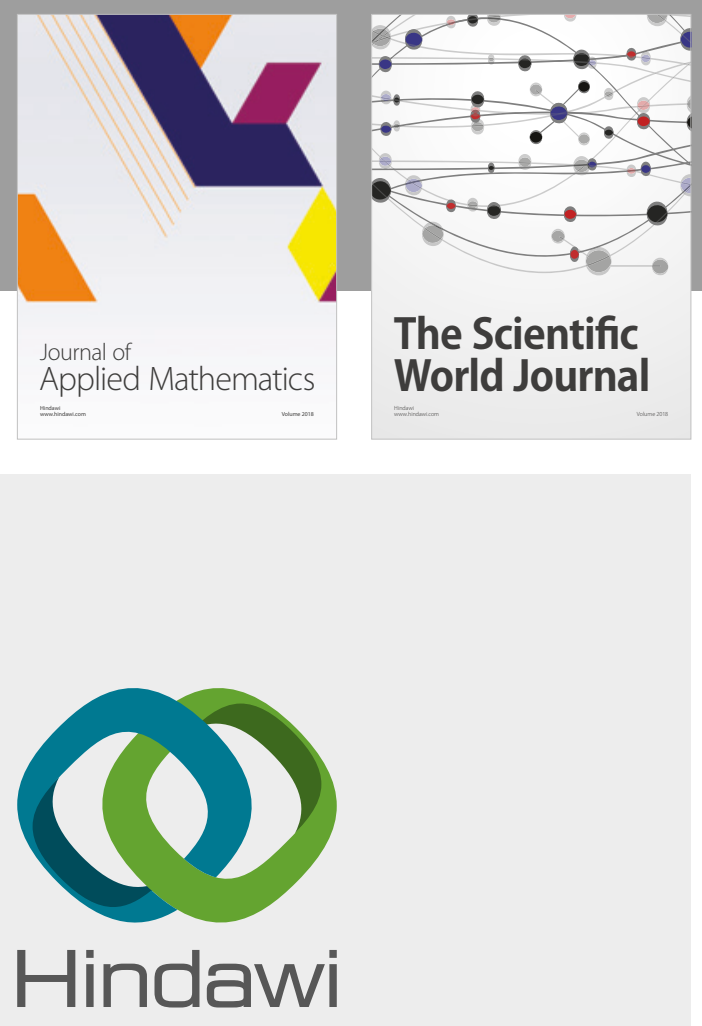

Submit your manuscripts at

www.hindawi.com

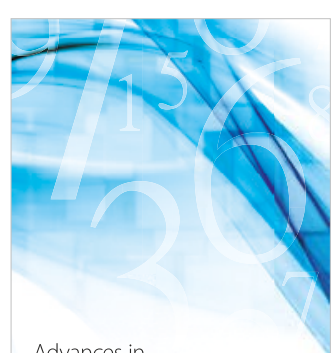

Advances in
Numerical Analysis
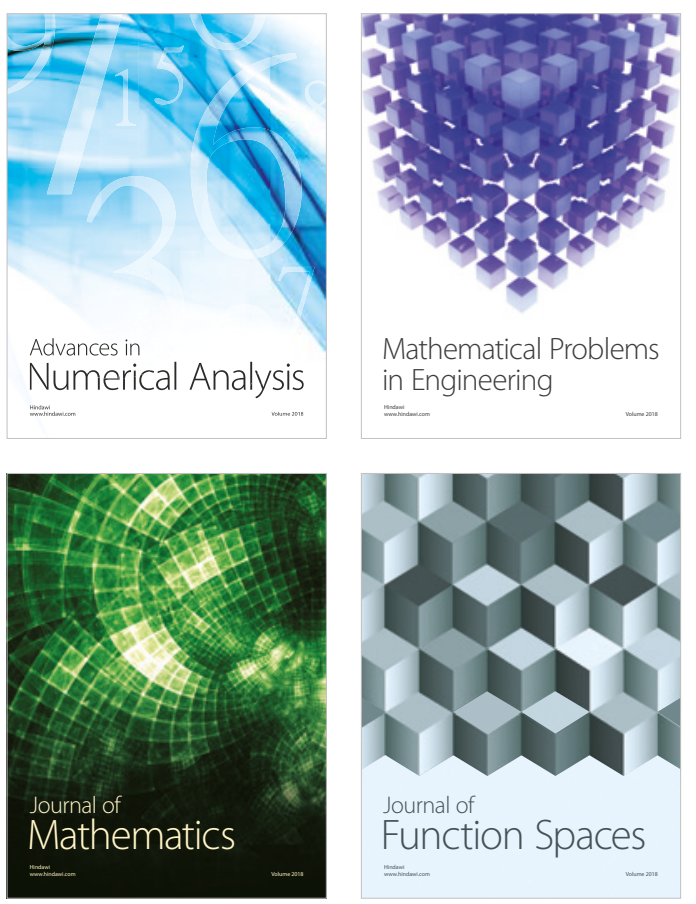

Mathematical Problems in Engineering

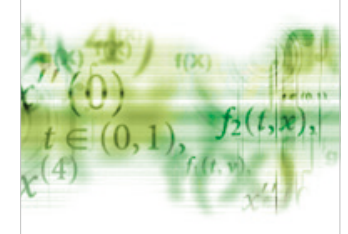

International Journal of

Differential Equations

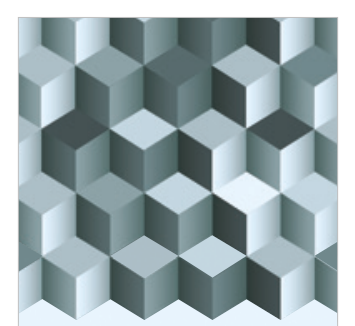

Journal of

Function Spaces

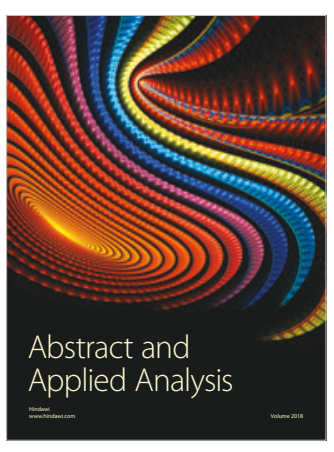

The Scientific

World Journal

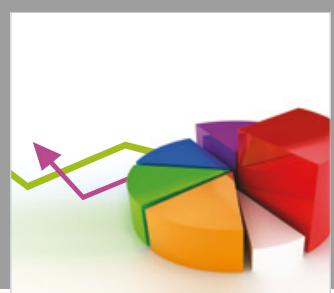

Journal of

Probability and Statistics
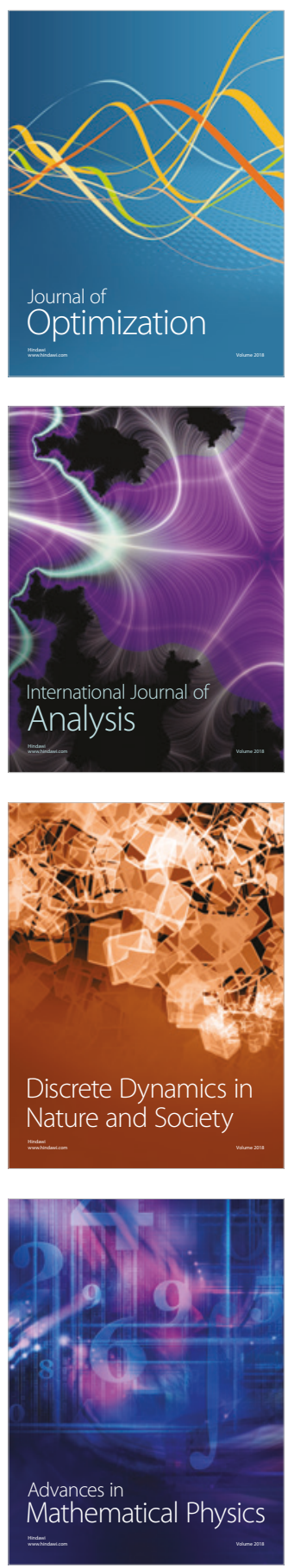